\title{
Selective intra-prediction Skip Algorithm for inter-frame coding in H.264/AVC
}

\author{
Byeongdu La $^{\text {a) }}$, Jinwoo Jeong ${ }^{\mathrm{b})}$, and Yoonsik Choe ${ }^{\mathrm{c})}$ \\ Department of Electrical and Electronic Engineering, Yonsei University, 134 \\ Sinchon-dong, Seodaemun-gu, Seoul, 120-749, Korea \\ a)bdla6077@yonsei.ac.kr \\ b) $691 @ y o n s e i . a c . k r$ \\ c)yschoe@yonsei.ac.kr
}

Abstract: Inter-frame coding using inter- and intra-predictions plays an important role in achieving high compression efficiency in H.264/AVC. However, most intra-predictions are unnecessary, since the intra-coding mode occupies less than $5 \%$ of the overall coding in interframe coding. In this paper, we propose an intra mode skip algorithm for inter-frame coding in H.264/AVC as a means of obtaining fast intra mode decision. The algorithm uses the joint entropy (JE) and mutual information (MI) to extract the temporal correlations between the current block and the reconstructed block based on $8 \times 8$ motion estimation. All or part of the intra-prediction search is omitted by using JE and MI. Experimental results show that the proposed algorithm is capable of reducing the overall coding time by $15-37 \%$ and the overall intra-prediction time by $32-78 \%$ compared to full search of the reference software, with negligible loss of quality.

Keywords: H.264, intra prediction, entropy, mutual information

Classification: Electron devices, circuits, and systems

\section{References}

[1] Joint Video Team (JVT) of ISO/IEC MPEG and ITU-T VCEG, "Draft ITU-T recommendation and final draft international standard of joint video specification (ITU-T Rec. H.264 and ISO/IEC 14496-10 AVC)," March 2003.

[2] B. G. Kim, J. H. Kim, and C. S. Cho, "A Fast Intra Skip Detection Algorithm for H.264/AVC Video Encoding," ETRI Journal, vol. 28, no. 6, pp. 721-731, Dec. 2006.

[3] U. Mithun and P. Gupta, "An Early Intra Mode Skipping Technique for Inter Frame Coding in H.264 BP," Consumer Electronics, ICCE 2007, pp. 1-2, 10-14 Jan. 2007.

[4] Z. Cernekova, I. Pitas, and C. Nikou, "Information Theory-Based Shot Cut/Fade Detection and Video Summarization," IEEE Trans. Circuits Syst. Video Technol., vol. 16, pp. 82-91, Jan. 2006. 


\section{Introduction}

H.264/AVC [1] is the latest video coding standard developed by the Joint Video Team (JVT), which is an organization of the ISO Moving Picture Experts Group (MPEG) and the ITU-T Video Coding Experts Group (VCEG). Compared to the previous standards, H.264/AVC has many advanced characteristics such as $4 \times 4$ integer transformation, spatial intra-prediction, quarterpixel motion compensation, and so on. These added characteristics permit H.264/AVC to achieve higher coding efficiency than other standards, especially by using intra-prediction and variable block size motion compensation. To maximize the coding efficiency, H.264/AVC uses the rate distortion optimization (RDO) technique. The RDO method checks inter/intra-predictions for I/P-pictures to choose the best coding mode. The computational complexity is increased by using RDO calculation in all possible modes of interand intra-predictions.

To reduce this complexity, a number of fast algorithms for inter- and intra-predictions have been proposed. In this paper, we focus only on intraprediction. One of approaches to reducing complexity is based on the intraprediction skipping in the inter frame coding. Kim et al. proposed a fast intra-skip detection algorithm using the RD costs of neighboring MBs [2]. If the minimum RD cost of neighboring MBs is less than the sum of the absolute differences (SAD) for the current block in the best inter mode, intraprediction is executed. Otherwise intra-prediction is skipped. Mithun and Gupta proposed an early intra-mode skip technique that exploits temporal information extracted from the SAD value of the current frame MB and the reference frame MB [3]. Even though these algorithms reduce the complexity of intra-prediction, greater reduction is still possible because approximately $95 \%$ of intra-prediction is unnecessary in inter-frame coding.

In this paper, we propose a fast intra-prediction skip algorithm based on information theory. The joint entropy (JE) and mutual information (MI) are calculated from the current $16 \times 16$ block and the reconstructed $16 \times 16$ block based on $8 \times 8$ motion estimation from the reference frames. Using the JE and MI, the correlation between the two blocks and the image complexity of the two blocks can be measured. These measures can be used to set thresholds for selectively skipping the intra-prediction mode. Experimental results show that this fast intra-prediction skip algorithm significantly decreases the computational complexity.

In Section 2, we present an overview of the coding-mode decision in H.264/AVC. In Section 3, we introduce our fast intra-mode skip algorithm based on information theory. We present the performance evaluations of the algorithm in Section 4 and our conclusions are given in Section 5.

\section{Choosing the Inter-frame Coding Mode in H.264/AVC}

The coding mode in H.264/AVC divides into inter- and intra-predictions. To select the best inter-frame coding mode for an MB, inter- and intrapredictions are performed via the rate distortion optimization method. The 
mode that has the minimum RD cost is selected as the best coding mode. The intra-prediction is executed after the inter-prediction is performed (based on motion estimation and motion compensation). The intra-prediction relies on spatial correlation with adjacent blocks. For coding efficiency, the correlation between adjacent blocks is used to encode the prediction mode. The prediction for the current block is made by using the boundary pixels of previously decoded blocks above and to the left of the current block. To determine the best mode for intra-prediction in a block, the residual between the current block and its predicted block is transformed, quantized, entropy-coded, and reconstructed in accordance with the prediction modes. The mode that has the minimum RD cost among the prediction modes is selected as the best mode.

To choose the best mode for intra-prediction, the $4 \times 4$ luma blocks, $16 \times 16$ luma block, and $8 \times 8$ chroma block are searched according to their prediction modes. The number of modes for luma and chroma components in an MB is therefore given by Chroma $8 \times($ Luma $4 \times 16+$ Luma 16), where Chroma 8 , Luma 4, and Luma 16 are the number of prediction modes for $8 \times 8$ chroma, $4 \times 4$ luma (I4MB), and $16 \times 16$ luma (I16 MB), respectively. This means that the RDO calculation must be performed $4 \times(9 \times 16+4)=592$ times to select the best mode in an MB, which greatly increases the computational load. In inter-frame coding, the intra coding mode accounts for less than $5 \%$ of the overall coding on average. This means that most intra-predictions are unnecessary in inter-frame coding. Therefore, if intra-predictions can be effectively skipped in inter-frame coding, the computational complexity can be reduced dramatically.

\section{Proposed fast mode decision algorithm}

The detection of shot boundaries and key frames in a video sequence can be accomplished using information theory concepts such as the MI and JE between frames [4]. In H.264/AVC, the detection of shot boundaries and key frames can be understood as a process that extracts the temporal correlation between a $16 \times 16$ block of the current frame and a reconstructed $16 \times 16$ block based on motion estimation from the reference frames. Hence, information theory may provide an approach to skip the intra-predictions in inter-frame coding by using the temporal correlation between two blocks, as in the technique of Mithun and Gupta [3].

In determining the JE and MI between two blocks to extract the temporal correlation, a $16 \times 16$ block (denoted by $B L O C K_{O}$ ) of the current frame and a $16 \times 16$ block (denoted by $B L O C K_{R}$ ) reconstructed from the $8 \times 8$ inter mode are used. When the video levels of the two blocks vary from 0 to 255 , the video level transition between $B L O C K_{O}$ and $B L O C K_{R}$ can be measured by the transition probability as described by Cernekova et al. [4]:

$$
P_{O, R}(x, y), 0 \leq x \leq 255,0 \leq y \leq 255
$$

This is the probability that a pixel at level $x$ in $B L O C K_{O}$ has level $y$ in $B L O C K_{R}$. In other words, this is the number of pixels that change from 
level $x$ in $B L O C K_{O}$ to level $y$ in $B L O C K_{R}$ divided by the number of pixels in a block. Therefore, the JE (denoted by $H_{O, R}$ ) and MI (denoted by $I_{O, R}$ ) between $B L O C K_{O}$ and $B L O C K_{R}$ is given by

$$
\begin{aligned}
H_{O, R} & =-\sum_{x=0}^{255} \sum_{y=0}^{255} P_{O, R}(x, y) \log P_{O, R}(x, y) \\
I_{O, R} & =\sum_{x=0}^{255} \sum_{y=0}^{255} P_{O, R}(x, y) \log \frac{P_{O, R}(x, y)}{P_{O}(x) P_{R}(y)}
\end{aligned}
$$

To obtain the JE and MI distributions in video sequences for a $16 \times 16$ block using Eqs. (1) and (2), the 256 pixels may be coarsened to describe the JE and MI because the range of video levels is 256 . Thus, the range of video levels is reduced from $0-255$ to $0-63$ by dividing the range of levels by 4 . This division can be implemented by a simple shift operation. This change also reduces the complexity of calculating the JE and MI. Equations (1) and (2) can now be written as

$$
\begin{aligned}
H_{O, R} & =-\sum_{x=0}^{63} \sum_{y=0}^{63} P_{O, R}(x, y) \log P_{O, R}(x, y) \\
I_{O, R} & =\sum_{x=0}^{63} \sum_{y=0}^{63} P_{O, R}(x, y) \log \frac{P_{O, R}(x, y)}{P_{O}(x) P_{R}(y)}
\end{aligned}
$$

By applying Eqs. (3) and (4) to the a CIF video sequences in the JM10.1 reference software, the distribution of the resulting intra-coding modes (relative to the JE and MI) is shown in Fig. 1. In the figure, the probability of being the $4 \times 4$ intra mode is high as JE and MI increase when the nonhomogeneity of the two blocks increases. In contrast, when the two blocks are homogeneous, the probability of being the $16 \times 16$ intra mode increases as JE and MI decrease.

The JM reference software executes the intra-predictions after the interpredictions. This means that the JE and MI can be used to extract the temporal correlation and skip the intra-predictions. By using the distribution of Fig. 1, the threshold T1 is defined for the JE, and T2 is defined for the MI. If JE is less than T1, the intra-prediction for $4 \times 4$ blocks is skipped, and when MI is greater than T2, the intra-prediction for $16 \times 16$ block is skipped. When JE is less than T1 and MI is greater than T2 (the overlapped condition), the complete intra-prediction is skipped because of their relatively minor effect on the RD performance. The threshold values $\mathrm{T} 1$ and $\mathrm{T} 2$ can be changed in accordance with the desired performance. As the value of $\mathrm{T} 1$ increases, the portion that searches for the $4 \times 4$ blocks will decrease, resulting in a degradation of visual quality while saving time. By decreasing the value of $\mathrm{T} 2$, the portion that searches for $16 \times 16$ block decreases and results in a degradation of visual quality while saving time. In this paper, we set the threshold at 1.8 for $\mathrm{T} 1$ and 0.1 for $\mathrm{T} 2$. These values were determined according to experiments with a variety of video sequences based on the distribution of JE and MI. 


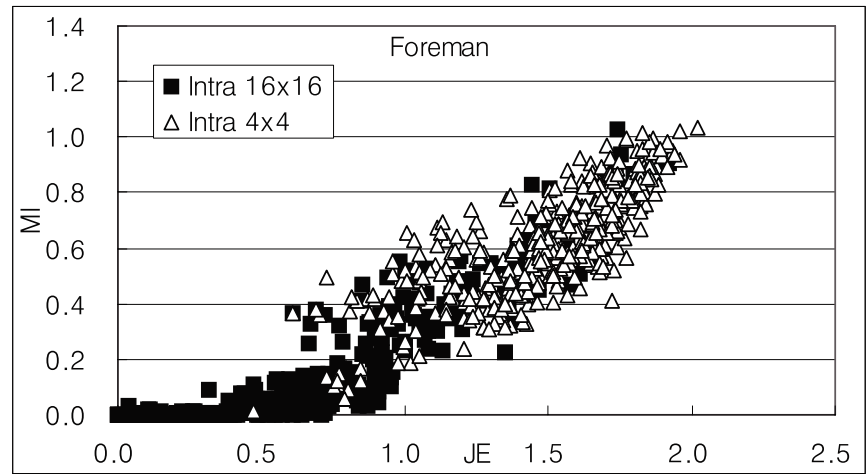

Fig. 1. Distribution of the intra $4 \times 4$ and $16 \times 16$ coding mode relative to the $\mathrm{JE}$ and $\mathrm{MI}$ of $\mathrm{CIF}$ sequence (IPPP type, Frame rate: 30, QP: 28).

\section{Performance evaluations}

For evaluation, the proposed algorithm, the previously proposed Kim et al.'s algorithm [2], the previously proposed Mithun et al.'s algorithm [3], and the full search were simulated on QCIF and CIF sequences in the JM10.1 reference software. And all algorithms are compared to the full search. All of the test sequences were coded in IPPP sequence type for quantization value 28, and one hundred frames were coded in every sequence. In the Table I, a negative value of $\Delta \mathrm{T}_{-} \mathrm{E}$ and $\Delta \mathrm{T}_{-} \mathrm{I}$ indicates the percent of time saved during the entire coding and entire intra-prediction compared to the full search by each. $\triangle$ PSNR is the value of PSNR that is changed compared to that of the full search and $\Delta$ Bit indicates the percent of bit changed compared to that of the full search.

As shown in Table I, For the proposed algorithm, the entire coding time and entire intra-prediction time of QCIF sequences for the IPPP sequence type on average was reduced by $31 \%$ and $65 \%$, respectively, while the PSNR and bit rate changed by $-0.01 \mathrm{~dB}$ and $0.9 \%$, respectively. For the "Foreman" and "Akiyo" of CIF sequences, about $80 \%$ of the time saving was achieved on intra-prediction, while maintaining almost the same level of PSNR and bit rate compared to the full search. The merit of our algorithm mainly comes from selecting the inter mode on the overlapped condition as previously described while discriminating the block that must be coded with $4 \times 4$ or $16 \times 16$ intra mode. Kim et al.'s algorithm [2] achieves a slightly better speed than other algorithms for a "Mobile" sequence that has large motion. However, the time of this algorithm on average was reduced by only $10-16 \%$ for the entire coding and $21-31 \%$ for the intra-prediction. For the Mithun and Gupta algorithm [3], even though the entire intra-prediction time on average was reduced by about $50 \%$, our proposed algorithm outperforms this algorithm in the time saving. Figure 2 shows the RD curve for the QCIF "News" sequence and the CIF "Mobile" sequence with QP values of 20, 24, 28,32 , and 36 . These results indicate that the proposed algorithm maintains the RD performance of the full search with slight loss of quality or increase in bitrate. 
Table I. Evaluation of the QCIF/CIF sequence (IPPP type).

\begin{tabular}{|c|c|c|c|c|c|c|c|c|c|c|c|c|c|}
\hline \multirow[t]{2}{*}{ Res. } & \multirow[t]{2}{*}{ Sequence } & \multicolumn{4}{|c|}{ Kim et al. [2] } & \multicolumn{4}{|c|}{ Mithun et al. [3] } & \multicolumn{4}{|c|}{ Proposed } \\
\hline & & $\Delta \mathrm{T}_{-} \mathrm{E}$ & $\Delta \mathrm{T}_{-} \mathrm{I}$ & $\triangle \mathrm{PSNR}$ & $\Delta \mathrm{Bit}$ & $\Delta \mathrm{T}_{-} \mathrm{E}$ & $\Delta \mathrm{T}_{-} \mathrm{I}$ & $\triangle \mathrm{PSNR}$ & $\Delta \mathrm{Bit}$ & $\Delta \mathrm{T} \_\mathrm{E}$ & $\Delta \mathrm{T}_{-} \mathrm{I}$ & $\triangle \mathrm{PSNR}$ & $\Delta \mathrm{Bit}$ \\
\hline \multirow{7}{*}{ QCIF } & News & -6.6 & -13.6 & -0.01 & 1.1 & -28.5 & -49.6 & 0.00 & 0.0 & -31.8 & -63.7 & -0.02 & 1.8 \\
\hline & Container & -8.0 & -16.5 & 0.00 & 0.0 & -24.7 & -48.5 & 0.00 & 0.0 & -27.2 & -56.3 & 0.00 & 0.3 \\
\hline & Foreman & -11.3 & -25.2 & 0.00 & -0.1 & -28.5 & -49.1 & 0.01 & 0.4 & -31.4 & -63.1 & 0.02 & 0.3 \\
\hline & Salesman & -11.4 & -22.9 & 0.00 & 0.1 & -28.7 & -48.6 & 0.00 & 0.0 & -29.1 & -58.0 & -0.02 & -0.2 \\
\hline & Table tennis & -11.5 & -28.5 & -0.01 & 1.3 & -27.4 & -47.8 & 0.00 & 0.0 & -32.3 & -71.0 & -0.05 & 3.1 \\
\hline & Akiyo & -8.7 & -22.0 & 0.00 & 0.0 & -28.5 & -51.4 & 0.00 & 0.0 & -33.8 & -74.6 & 0.00 & 0.0 \\
\hline & Average & -9.6 & -21.4 & 0.00 & 0.4 & -27.7 & -49.2 & 0.00 & 0.1 & -30.9 & -64.5 & -0.01 & 0.9 \\
\hline \multirow{7}{*}{ CIF } & Paris & -10.4 & -19.7 & -0.01 & 0.1 & -26.2 & -48.9 & (20.00 & -0.1 & -27.0 & -50.4 & -0.01 & (20.0 \\
\hline & Mobile & -30.0 & -56.5 & 0.00 & 0.0 & -28.1 & -49.2 & 0.00 & 0.0 & -17.9 & -32.1 & 0.00 & 0.1 \\
\hline & Salesman & -10.4 & -21.1 & 0.00 & 0.1 & -24.7 & -47.7 & 0.00 & 0.0 & -34.0 & -68.5 & -0.01 & 0.8 \\
\hline & Foreman & -11.9 & -26.9 & -0.02 & -0.1 & -24.6 & -49.9 & -0.02 & -0.2 & -36.6 & -76.4 & -0.04 & 0.4 \\
\hline & Tempete & -21.9 & -41.6 & -0.02 & 0.6 & -26.5 & -49.2 & -0.01 & 0.0 & -20.1 & -37.9 & -0.02 & 0.5 \\
\hline & Akiyo & -9.6 & -19.9 & 0.00 & -0.1 & -26.8 & -56.8 & 0.00 & 0.2 & -36.7 & -77.9 & 0.00 & -0.1 \\
\hline & Average & -15.7 & -30.9 & -0.01 & 0.1 & -26.2 & -50.3 & 0.00 & 0.0 & -28.7 & -57.2 & -0.01 & 0.3 \\
\hline
\end{tabular}
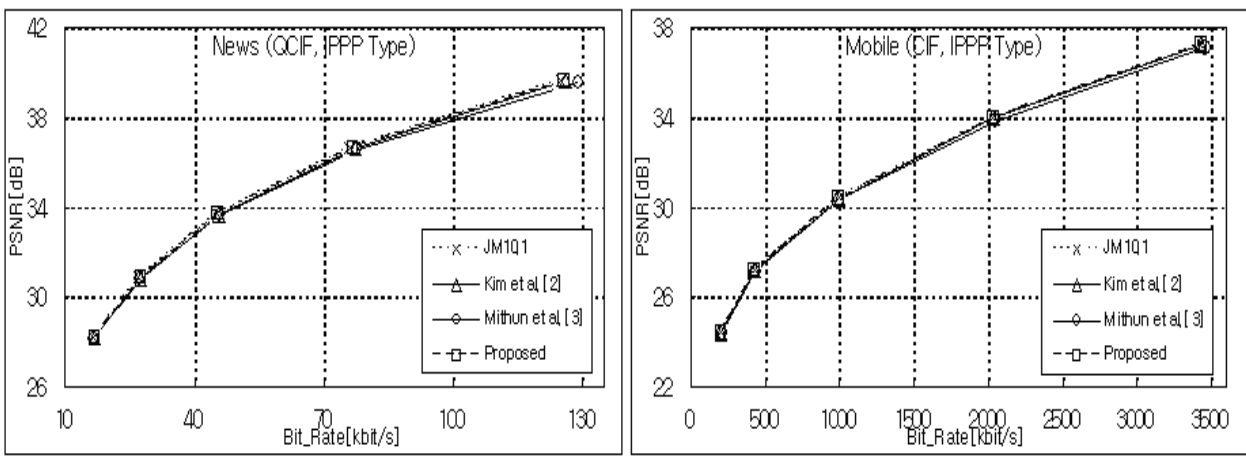

Fig. 2. RD curve for the QCIF "News" sequence and CIF "Mobile" sequence.

\section{Conclusions}

In this paper, we introduced a fast intra-prediction skip algorithm based on information theory for H.264/AVC. The JE and MI between the current block and the block reconstructed from $8 \times 8$ motion estimation were calculated. The coding time was reduced using these values as an intra-prediction skipping threshold. The thresholds can be applied selectively to determine whether to skip the $4 \times 4$ mode, the $16 \times 16$ mode, or the entire intra mode by using the value of JE and MI. The results demonstrate that the proposed algorithm reduces the entire intra-prediction time by $32-78 \%$ compared to that of the full search, with only a negligible loss of quality and increase in bit rate. This algorithm is suitable for software application than hardware with their additional calculation for JE and MI.

\section{Acknowledgments}

This research was supported by the MKE (Ministry of Knowledge Economy), Korea, under the ITRC (Information Technology Research Center) support program supervised by the IITA (Institute of Information Technology Assessment) (IITA-2008-(C1090-0801-0011)). 